\section{Marker für pulmonale Langerhans-Zell Histiozytose}

Lommatzsch $\mathrm{M}$ et al. Bronchoalveolar lavage for the diagnosis of pulmonary Langerhans cell histiocytosis. Respir Med 2016; 119: 168 - 174

Die pulmonale Langerhans-Zell Histiozytose $(\mathrm{PLZH})$ ist eine seltene $\mathrm{Er}$ krankung, deren definitive Diagnose eine Lungenbiopsie erfordert. Die ist aber invasiv und kann zu Komplikationen führen. Bei typischen klinischen Befunden wird von Leitlinien daher auch der Nachweis CD1a-positiver Zellen in der broncho-alveolären Lavage empfohlen. Da dies aber eine schlechte Sensitivität und Spezifität aufweist, wäre eine verbesserte Zellanalyse wünschenswert.

Aufnahme in die Studie fanden konsekutive Patienten mit neu diagnostizierter $\mathrm{PLZH}$, Einschlusskriterien waren ein charakteristischer $\mathrm{CT}$-Befund und ein aktueller Nikotinkonsum von mindestens 20 Zigaretten pro Tag. Ausschlusskriterien waren eine Behandlung mit inhalativen oder systemischen Immunsuppressiva sowie die frühere Diagnose einer PLZH. Als Kontrollen dienten asymptomatische Niemalsraucher mit normaler Lungenfunktion ( $n=21$ ) sowie asymptomatische Raucher mit einem Nikotinkonsum von mindestens 10 Zigaretten täglich und 10 Packyears $(n=40)$. Bei den Teilnehmern wurden mittels Vierfarben-Durchflusszytometrie in der broncho-alveolären Lavage plasmazytoide und myeloide dendritische Zellen bestimmt.

Eingang in die Analyse fanden neben den Kontrollen 10 Patienten mit neu diagnostizierter PLZH. Bei 5 Patienten war die Diagnose histologisch bestätigt worden, bei den 5 anderen basierte die Diagnose auf einem typischen CT-Befund sowie einer typischen Krankengeschichte. In der broncho-alveolären Lavage zeigte sich bei den Patienten ein medianer Anteil von 3,6\% CD1a-positiver Zellen und lag damit eher niedriger als in den beiden Kontrollgruppen. Im Vergleich zu den Kontrollen zeigten Patienten mit
PLZH auch keine erhöhte Rate an myeloiden dendritischen Zellen (Median 0,79\% der Leukozyten in der Lavage) und deren Subpopulation Langerhans-Zellen (Median $0,44 \%$ ). Auch fand sich bei ihnen eine normale Expression des Reifemarkers CD83 auf den myeloiden dendritischen Zellen in der Lavage. Allerdings war die Expression von CD80 auf den myeloiden dendritischen Zellen bei den Patienten signifikant niedriger als in beiden Kontrollgruppen mit Abhängigkeit von der Schwere der Erkrankung. In der Analyse der ROC (Receiver Operating Characteristic)-Kurve fand sich ein Anteil von 53\% CD80-positiver myeloider dendritischer Zellen in der broncho-alveolären Lavage als optimaler Grenzwert für die Diagnose einer PLZH mit einer Sensitivität und Spezifität von jeweils 0,90 .

FAZIT

Bei Patienten mit PLZH fand sich kein erhöhter Anteil an Langerhans-Zellen in der broncho-alveolären Lavage, allerdings war bei ihnen der Anteil an CD80-positiven myeloiden dendritischen Zellen erniedrigt. Aufgrund der höheren Sensitivität und Spezifität erscheint dieser Marker nach Ansicht der Autoren daher geeigneter für die Diagnose einer PLZH als der bisher empfohlene Marker CD1a.

Dr. med. Johannes Weiß, Bad Kissingen 